NASA Technical Memorandum 83658

NASA-TM-83658

19840016951

\title{
Optimization of Fringe-Type Laser Anemometers for Turbine Engine Component Testing
}

Richard G. Seasholtz, Lawrence G. Oberle, and Donald H. Weikle Lewis Research Center Cleveland, Ohio

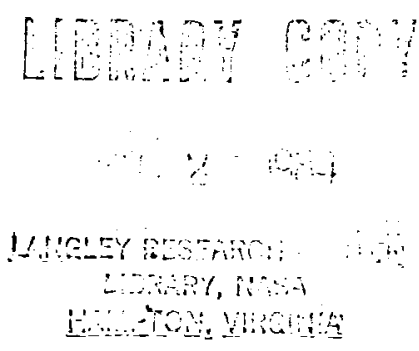

Prepared for the

Twentieth Joint Propulsion Conference cosponsored by the AIAA, SAE, and ASME Cincinnati, Ohio, June 11-13, 1984 
$\because$

"

$\cdots$ 


\section{And}

AIAA-84-1459

Optimization of Fringe-Type Laser

Anemometers for Turbine Engine

Component Testing

Richard G. Seasholtz, Lawrence G. Oberle

and Donald $\mathrm{H}$. Weikle, Lewis Research Center

Cleveland, $\mathrm{OH}$

\section{AIAA/SAE/ASME 20th Joint Propulsion Conference June 11-13, 1984/Cincinnati, Ohio}


$\because$

$\because$ 
OPTIMIZATION OF FRINGE-TYPE LASER ANEMOMETERS FOR TURBINE ENGINE COMPONENT TESTING

Richard G. Seasholtz, Lawrence G. Oberle, and Donald H. Weikle National Aeronautics and Space Administration

Lewis Research Center

Cleveland, Ohio 44135

\section{Abstract}

The fringe-type laser anemometer is analyzed using the Cramer-Rao bound for the variance of the estimate of the Doppler frequency as a figure-ofmerit. Mie scattering theory is used to calculate the Doppler signal wherein both the amplitude and phase of the scattered light are taken into account. The noise from wall scatter is calculated using the wall bidirectional reflectivity and the irradiance of the incident beams. A procedure is described to determine the optimum aperture mask for the probe volume located a given distance from a wall. The expected performance of counter-type processors is also discussed in relation to the Cramer-Rao bound. Numerical examples are presented for a coaxial backscatter anemometer.

\section{Nomenclature}

a

A

C

$<>$

E

$\mathrm{E}_{0}$

$f_{B}$

$f_{D}$

F

h

$I(x, y)$

$\mathrm{k}$

$\stackrel{k}{\sim} 1,2$

$\mathrm{k}_{\mathrm{s}}$

$\mathrm{L} r$

$\mathrm{L}_{b}$

$\stackrel{n}{\sim}$

$n_{i}$

$n_{0}$

$n(x, y)$

$n_{k}$

$N_{f}$

$N_{0}$ expected peak pedestal counts per

$$
\text { Doppler cycle }
$$

expected peak pedestal counts per second

velocity of light

expected value of

electric field vectors

electric field amplitude

frequency difference between incident

$$
\text { beams, } \mathrm{Hz}
$$

Doppler frequency, $\mathrm{Hz}$

lens focal length

Planck's constant

irradiance at point $(x, y)$

wave number $(=|\underline{k}|)$

wave vectors of incident beams

wave vector of scattered light

radiance of laser light reflected

by wall

radiance of background light

measurement set vector

number counts in $i^{\text {th }}$ time interval

expected noise, counts per Doppler cycle

noise defined by eq. (10)

noise counts due to flux through $k$ th

area element (eq. (21))

number fringes between $1 / \mathrm{e}^{2}$ irradiance

points

expected value of noise from wall scatter and background $\hat{p}_{1,2}$

$p(x, y)$

$p(q, n)$

$p_{0}$

$P_{\ell}$

$\hat{q}_{1,2}^{\ell}$

$q_{1,2}$

$q(x, y)$

$q_{0}$

$q_{k}$

$Q$

R

$\hat{s}_{1,2}$

$s(x, y)$

so

$s_{k}$

$S_{\alpha}(\theta)$

SNR

$t$

$t_{0}$

v

v)

w

$w_{0}$

$z_{0}$

$\alpha_{i}$

B

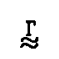

$\delta_{\alpha}(\theta)$

$\delta_{\alpha \beta}$

$\Delta A$

$\Delta f$

$\Delta f$

$\Delta t$

$n$

$\theta$ unit vectors normal to incident beams and in scattering planes

pedestal signal defined by eq. (11)

likelihood function

integral of $p(x, y)$ over aperture

laser power

unit vectors normal to scattered beam and in scattering planes

signal defined by eq. (11)

integral of $q(x, y)$ over aperture

signal counts due to flux through $k$ th area element (eq. (21))

quality factor defined by eq. (22)

distance from scatterer to observation point

unit vectors normal to scattering planes

signal defined by eq. (11)

integral of $s(x, y)$ over aperture

signal counts due to flux through $k$ th area element (eq. (21))

Mie scattering amplitude function

$(\alpha=$ "s", "p")

signal-to-noise ratio

time, sec

time at peak of Doppler burst, sec visibility of Doppler burst

variance of ( )

beam radius at $1 / e^{2}$ irradiance point

beam waist radius

intrinsic impedance of free space

$i^{\text {th }}$ unknown parameter

half of beam crossing angle

Fisher information matrix

phase of scattering light ( $\alpha=$ "s", "p")

phase difference of light scattered from

two beams (eq. (B13))

area element in aperture plane

system noise bandwidth

time interval

quantum efficiency

angular coordinate

This paper Is declared a work of the U.S. 


\begin{tabular}{|c|c|}
\hline$\theta_{1,2}$ & scattering angles \\
\hline$\lambda$ & wavelength of laser light \\
\hline$\rho^{\prime}$ & bidirectional reflectivity \\
\hline$\sigma_{\alpha \beta}$ & $\begin{array}{l}\text { scattering cross sections defined by } \\
\text { eq. (B13) }\end{array}$ \\
\hline${ }^{\sigma} \mathrm{fD}$ & $\begin{array}{l}\text { standard deviation of estimate of } \\
\text { Doppler frequency }\end{array}$ \\
\hline$\phi$ & angular coordinate \\
\hline$\phi_{\mathrm{f}}$ & $\begin{array}{l}\text { flow angle relative to fringe normals } \\
\text { phase of Doppler signal }\end{array}$ \\
\hline$\Omega^{\prime}$ & $\begin{array}{l}\text { solid angle subtended by pinhole image at } \\
\text { point in aperture planel }\end{array}$ \\
\hline
\end{tabular}

\section{Introduction}

The laser anemometer (LA) has become a valuable tool in turbine engine research, providing data that would be almost impossible to gather using conventional instrumentation. However, this application of LA has proven to be one of its more difficult. Measurements in turbomachinery components are typified by small passages and highly accelerated, high velocity flows. This leads to the requirement for small seed particles that will faithfully follow the flow. Unfortunately, small particles are weak light scatterers, which result in low signal levels. When coupled with the high level of noise caused by laser light scattered from surfaces near the measurement point, the signal-to-noise ratio (SNR) is often less than needed for accurate determination of the particle velocity.

Limitations of the conventional dual-beam fringe-type LA have forced researchers to look for new techniques and new optical designs.

In particular, the dual-focus (two-spot) LA was developed $(1)$ for use in engine research. This system is more sensitive to small particles and has better stray light rejection properties than the fringe LA. However, the dual-focus LA generally is more expensive and requires more sophisticated signal processing than the fringe LA. Also, in highly turbulent flows, the data rate tends to be lower than the data rate achieved with the fringe LA. (This low data problem may be solved by use of elliptical spot systems such as described in ref. 2.)

One technique that has permitted the fringe LA to be successfully used in compressor and turbine testing is the use of fluorescent seed particles. (3-6) The laser-induced fluorescence from the particles is separated from the laser light scattered from walls by an optical filter. This allows measurements to be made close to surfaces. Unfortunately, the fluorescent efficiency of the dyes used decreases rapidly as the temperature of the flow increases above room temperature. Thus, unless a high efficiency, high temperature fluorescent seed material is found, it appears that this technique will not be usable for high temper-
ature flows.

Another approach $(7,8)$ for making measurements close to walls with the fringe LA is based on using the differences of the polarization properties of the light scattered from particles and from solid surfaces. However, the success of this technique depends on the assumption of nonabsorbing spherical particles and on the details of the surface, such as roughness and the presence of deposits.

In spite of its limitations, we feel that the fringe LA is suitable for many turbine engine component tests. For this reason, a detailed analysis of the fringe LA was conducted with the purpose of defining its limits of applicability. This paper addresses the problem of optimizing the optical system to achieve the best measurement of the velocity of a single seed particle. Note that this is only one part of the overall problem of the rapid, accurate determination of the flow parameters in turbine engine components. 0ther factors must also be considered, such as statistical biasing and proper data reduction methods.

In this paper, the fringe-type laser anemometer is analyzed using the Cramer-Rao bound for the variance of the estimate of the Doppler frequency as a figure-of-merit. The noise from wall scatter is calculated using the wall bidirectional reflectivity and the irradiance of the incident beams. Mie scattering theory is used to calculate the Doppler signal.

Similar work has been reported $(9,10)$ for evaluating the signal-to-noise ratio for measurements close to walls. That work is extended here by treating the particle scattering using Mie scattering theory (instead of assuming a constant cross section) and by characterizing the wall reflectivity by the bidirectional reflectivity (instead of assuming a constant value for the reflectivity). Thus the analys is presented here can be used to evaluate the effect of various wall treatments and coatings which have reflectivities that are a function of both the incident light direction and the observation direction.

In addition, a procedure is presented for determining the optimum aperture mask for the probe volume located a given distance from a wall. The use of circular aperture masks to decrease probe volume length has been recognized(11) and used in LA systems for compressor and turbine studies (3-6). However, the procedure presented here determines the optimum mask, which is not necessarily circular.

Because the counter-type processor is usually used with fringe LA's for high velocity flows, the relation between the Cramer-Rao bound and the required signal-to-noise ratio for counters is discussed.

Finally, numerical examples for a coaxial backscatter LA are presented to illustrate the procedure for determining the optimum aperture mask.

\section{Theory}

In this section the Cramer-Rao lower bound for the variance of the estimate of the Doppler frequency will be obtained in terms of the parameters of the Doppler burst. The parameters related to the signal will be determined using Mie scattering theory. The noise parameter will be determined 
by evaluating the laser light scattered by walls located near the probe volume. Finally, the performance of the counter-processor will be related to the Cramer-Rao bound.

\section{Doppler Burst Signal}

A particle passing through the probe volume of a dual-beam fringe-type laser anemometer (fig. 1) with the indicated trajectory (described by $\delta$, the distance of closest approach to the probe volume axis, and by $\phi f$, the angle between the flow direction and the fringe normals) will generate a Doppler burst signal (assumed to be detected with a photomultiplier tube)

$\left\langle n_{k}\right\rangle=A e^{-2\left[\frac{2 f_{D}\left(t_{k}-t_{0}\right)}{N_{f} \cos \phi_{f}}\right]^{2}}$
$\left\{1+V \cos \left[2 \pi\left(f_{D}+f_{B}\right)\left(t_{k}-t_{0}\right)+\psi\right]\right\} \Delta t+N_{0} \Delta t$

where $\left\langle n_{k}\right\rangle$ is the expected number of photoelectron counts in the time interval $\left(t_{k}-\Delta t / 2\right.$, $\left.t_{k}+\Delta t / 2\right), t_{0}$ is the time at the peak of the Gaussian envelope, $f_{D}$ is the "Doppler" frequency, $f_{B}$ is the frequency difference between the two incident beams, $V$ is the visibility, $N_{0}$ is the constant background noise, $N_{f}=2 w_{0} / \mathrm{s}$ is the number of fringes, ( $w_{0}$ is the waist radius of the incident beams and $s$ is the fringe spacing) and

$$
A=A_{0} e^{-2\left(\delta / w_{0}\right)^{2}}
$$

is the peak value of the pedestal, where $A_{0}$ is the peak value if the trajectory passes through the center of the probe volume. The trajectory is assumed to be near the focal plane and to have no significant component along the optical axis. The particle is assumed to have constant velocity as it passes through the probe volume.

\section{Cramer-Rao Lower Bound}

The unknown parameters in the Doppler burst $c$ an be denoted by the vector $g=\left(f D, \phi f, t_{g}, A\right.$, $\left.\psi, N_{0}, V\right)$. The measurement can be described by the vector $n=\left(n_{1}, n_{2}, n_{3}, \ldots, n_{k}\right)$ with elements that are the photoelectron counts for $k$ equal time intervals $\Delta t$.

The Cramer-Rao lower bound for the yariance of the estimate of the parameter $\hat{\alpha}_{i}$ is $(12)$

$$
f\left(\hat{\alpha}_{i}\right)=\left(\underline{I}^{-1}\right)_{i}
$$

where $I$ is the Fisher information matrix with elements given by

$$
\Gamma_{i j}=-\left\langle\frac{a^{2} \ln p(\underline{n} \mid \alpha)}{\partial \alpha_{i} \partial \alpha_{j}}\right\rangle
$$

The conditional probability function $p(n ! \alpha)$ is the likelihood function, which expresses the probability that given a set of parameters $\underset{\alpha}{ }$, the set of observations $n$ will occur. (The maximum likelihood estimate for the parameters $a$ is given by the $\alpha$ that maximizes $p(n ı \alpha)$ for a given set of measurements $\underset{\sim}{n}$.)

For Poisson statistics, the probability of $n_{k}$ counts in one time interval is

$$
p\left(n_{k}\right)=e^{-\left\langle n_{k}\right\rangle}\left\langle n_{k}\right\rangle^{n_{k}} / n_{k}:
$$

where the expected value $\left\langle n_{k}\right\rangle$ is given by Eq. (1). The elements of Fisher information matrix can then be written (13)

$$
r_{i j}=\sum_{k} \frac{1}{\left\langle n_{k}\right\rangle} \frac{\partial\left\langle n_{k}\right\rangle}{\partial \alpha_{i}} \frac{\partial\left\langle n_{k}\right\rangle}{\partial \alpha_{j}}
$$

If the parameters are uncorrelated, the variance of the estimate of one of the parameters is given simply by the inverse of the corresponding diagonal element the Fisher information matrix; i.e., by the variance that would apply if only one parameter were unknown.

$$
\mathscr{V}\left(\hat{\alpha}_{i}\right) \geq 1 / \Gamma_{i j}
$$

Here, we assume that the parameters are only weakly correlated, so that this much simpler relation for the Cramer-Rao bound may be used.

If we assume that the background noise is greater than the peak signal, and that the number of fringes is large compared to one, we can let $\Delta t \rightarrow 0$ and convert the sum in Eq. (6) to an integral, which can be evaluated to give a lower bound for the variance of the estimate of the Doppler frequency

$$
\psi\left(\hat{f}_{D}\right) \geq \frac{64}{\pi 5 / 2} \frac{N_{0} f^{3}}{v^{2} A^{2} N^{3}}
$$

where we let $N=N_{f} \cos \phi_{f} ; i . e, N$ is the number of cycles between the $1 / \mathrm{e}^{2}$ times of the envelope of the burst. Note that the lower bound is not dependent on the frequency shift $f_{B}$. Equation (8) can be rewritten to give a lower bound for the relative error (corresponding to the Cramer-Rao bound) of measurements of the Doppler frequency

$$
\left(\frac{{ }^{\sigma_{D}}}{f_{D}}\right)=\frac{8}{\pi / 4} \frac{n_{0}^{1 / 2}}{a V N^{3 / 2}}
$$

where $\sigma_{f_{D}}=\left[\mathscr{V}\left(f_{D}\right)\right] 1 / 2$ and where $n_{0}=N_{0} / f_{D}$ and $a=A / f_{D}$ are the expected noise and signal counts per Doppler period.

A comparison (for a particular set of parameters) between a numerical evaluation of $\left(\sigma_{f_{D}} / f_{D}\right)$ using the full Fisher information matrix $\left(\mathrm{eq}^{\mathrm{D}}(6)\right)$, and the analytical expression (eq. (9)), is shown in Fig. 2. This indicates that the assumptions used in the derivation of Eq. (9) are valid for $n_{0} / a V>2$. The simple expression for the error bound (eq. (9)) will be used in the remainder of this paper.

The task now is -- given a particular optical configuration, seed particle, test environment, and flow -- to calculate the signal and noise. We 
then can use the lower bound of the relative error given by Eq. (9) as a figure-of-merit for this particular optical configuration. The optical configuration may then be optimized to find the maximum value of the lower bound (i.e., the smallest error in the measurement).

Noise

The background noise caused by laser light scattered from surfaces near the probe volume is a function of the optical configuration, the reflectance properties of the wall, and the distance of the wall from the probe volume. In addition to this direct wall scatter, there generally will also be a constant background noise flux caused by multiple reflections of the laser light, ambient

light, and blackbody radiation from hot surfaces.

The light flux through area $d A$ at a point $(x, y)$ on the aperture plane due to wall scatter and background radiance is obtained in Appendix $A$ by integrating the radiance $L$ of the wall over the solid angle subtended by the image of the pinhole at the point on the aperture (fig. 3). This light flux, expressed in terms of photoelectron counts per Doppler cycle is (eq. Al)

$n(x, y) d A=$

$\left(\frac{n \lambda}{h c f_{D}}\right)_{\Omega}\left[L_{r}\left(x, y ; \theta_{r}, \phi_{r}\right)+L_{b}\right] d \Omega \cos \theta d A$

For purposes of calculation, the aperture plane is assumed to be at the plane of the focusing lens instead of the position shown in Fig. 1 (the mask will have the same effect in either iocation). Also, for this calculation, we treat the laser light as if it were incoherent. This means that interference effects, such as speckle, are not taken into account. But the results obtained do represent the expected values.

\section{Signal}

For the geometry shown in Fig. 4 , the signal is calculated in Appendix $B$ using Mie scattering theory. The light flux through differential area element $d A$ located in the aperture plane at position $(x, y)$ consists of the following: the quadrature components of the Doppler modulated signal, $s(x, y)$ and $q(x, y)$; and the pedestal component, $p(x, y)$. These are expressed in terms of the number of counts/Doppler period as

$$
\begin{aligned}
& s(x, y) d A=\left(2 I_{0} / F^{2}\right)\left(n \lambda / h c f_{D}\right) \cos ^{3} \theta \sum_{\alpha, \beta} \sigma_{\alpha \beta} \cos \delta_{\alpha \beta} d A \\
& q(x, y) d A=\left(2 I_{0} / F^{2}\right)\left(n \lambda / h c f_{0}\right) \cos ^{3} \theta \sum_{\alpha, \beta} \sigma_{\sigma \beta} \sin \delta_{\alpha \beta} d A \\
& p(x, y) d A=\left(2 I_{0} / F^{2}\right)\left(n \lambda / h c f_{D}\right) \cos ^{3} \theta I_{s d c}
\end{aligned}
$$

The calculation takes into account both the variation in the amplitude and in the phase of the scattered fields as a function of scattering angle. Examples of the variation of the differential scattering cross section and phase angles are shown in Fig. 5 for $0.5,1.0$, and $1.5 \mu \mathrm{m}$ diameter polystyrene latex particles (refractive index $=1.59$ ) The figure shows that for near backscatter the " $p$ " polarized scattering is stronger than the " $\mathrm{s}$ " polarized scattering. Also, it can be seen that the phase angle variation is much larger for the larger particle. This phase variation is important because it leads to a reduction in the visibility of the Doppler burst when the signal is integrated over the aperture.

\section{Evaluation of Cramer-Rao Lower Bound}

The relations derived above for noise and signal are now used to evaluate the relative error given by Eq. (9) for a given optical configuration, seed particle, and test environment.

By integrating over the clear aperture (not including light blocked by the aperture mask), we obtain the total number of counts for the two quadrature signals, the pedestal, and the background

$$
\begin{aligned}
& s_{0}=\int s(x, y) d A \\
& q_{0}=\int q(x, y) d A \\
& p_{0}=\int p(x, y) d A \\
& n_{0}=\int n(x, y) d A
\end{aligned}
$$

These quantities are identified with the corresponding quantities in Eq. (1) for the Doppler burst signal; i.e.,

$$
A / f_{D}=a=p_{0}
$$

is the pedestal component,

$$
A V / f_{D}=a V=\left(s_{0}^{2}+q_{0}^{2}\right)^{1 / 2}
$$

is the component modulated at the Doppler frequency,

$$
V=\left(s_{0}^{2}+q_{0}^{2}\right)^{1 / 2} / p_{0}
$$

is the visibility, and

$$
\psi=\tan ^{-1}\left(q_{0} / s_{0}\right)
$$

is the phase.

For the background noise dominated case (for which Eq. (9) is valid), the Cramer-Rao lower bound for uncertainty in the measurement of the Doppler frequency is thus

$$
\left(\frac{\sigma_{f_{D}}}{f_{D}}\right)=\frac{8}{\pi^{5 / 4} N^{3 / 2}}\left(\frac{n_{0}}{s_{0}^{2}+q_{0}^{2}}\right)^{1 / 2}
$$

\section{Counter-processor}

The above analysis gives a lower bound for the uncertainty in the measurement of the velocity of a single particle (eq. (17)). In practice, this error bound cannot actually be achieved. In particular, the counter-processor exhibits a threshold at a signal-to-noise ratio (SNR) of about $10 .(14)$ For $S N R>10$ the counter $c$ an be expected to give 
good results, but below this level the measurement error increases rapidly. As in Ref. 14, we define the SNR as the ratio of the mean signal power at the peak of the Doppler burst to the noise power due to wall scatter and background

$$
\operatorname{SNR}=\frac{(A V)^{2}}{4 N_{0} \Delta f}=\frac{s_{0}^{2}+q_{0}^{2}}{4 n_{0}\left(\Delta f / f_{D}\right)}
$$

where $\Delta f$ is the noise bandwidth. (The noise bandwidth is determined by the low-pass and highpass filters in the processor.)

Comparing this expression (eq. (18)) for the SNR with Eq. (17) allows us to express the SNR in terms of the relative error $\left(\sigma_{f_{D}} / f_{D}\right)$ given by Eq. (9).

$$
S N R=\frac{16}{\pi^{5 / 2} N^{3}}\left(\frac{f_{D}}{\Delta f}\right)\left(\frac{\sigma_{D}}{f_{D}}\right)^{-2}
$$

Even with SNR > 10, the counter will not provide measurements as accurate as indicated possible by the Cramer-Rao bound because of other error sources such as the finite clock frequency and the effectiveness of validation circuitry.

\section{Numerical Examples}

Two examples of calculating the Cramer-Rao bound and selecting the optimum aperture mask are presented in this section. The following parameters are used for both examples: the fluid velocity is $500 \mathrm{~m} / \mathrm{sec}$; the seed particles are polystyrene spheres with $1 \mu \mathrm{m}$ diameter and real refractive index equal 1.59; no Bragg shift is used; the flow is perpendicular to the fringes; the laser power equals $0.1 \mathrm{~W}$ at $0.5145 \mathrm{um}$ wavelength; the beams are linearly polarized with the electric vector normal to the plane formed by the beams (along the $y$ axis); and system efficiency including losses and PMT quantum efficiency is 10 percent. The wall is normal to the optical axis, is located $1 \mathrm{~mm}$ from the center of the probe volume, and is a Lambertian reflector with reflectivity equal $1 / \pi$.

For the first example, which represents a low performance optical configuration: the focusing lens has a $200 \mathrm{~mm}$ focal length and a $50 \mathrm{~mm}$ diameter ( $f / 4)$; the pinhole image diameter is set equal to $125 \mathrm{\mu m}$; the number of fringes is 12.5 ; the fringe spacing is $10 \mu \mathrm{m}$; and the uniform background radiance is $3.1 \times 10^{-5} \mathrm{~W} / \mathrm{mm}^{2} / \mathrm{sr}$.

For the second example, which represents a high performance optical configuration: the focusing lens has a $250 \mathrm{~mm}$ focal length and a $100 \mathrm{~mm}$ diameter ( $\mathrm{f} / 2.5)$; the pinhole image diameter is set equal to $70 \mu \mathrm{m}$; the number of fringes is 10 ; the fringe spacing is $5 \mu \mathrm{m}$; and the uniform background radiance is $3.8 \times 10^{-3} \mathrm{~W} / \mathrm{mm}^{2} / \mathrm{sr}$. Note that the background radiance is set higher for this example than it was for the first example. This was done to insure that the assumption that the noise is larger than the signal remains valid. Of course, this indicates a more severe environment.

For these numerical calculations, the the aperture plane is divided in 400 equal areas.
But, because of symmetry, only one quadrant need be considered with 100 area elements.

The error bound can be expressed in terms of these 100 elements as

$$
\left(\frac{\sigma_{f_{D}}}{f_{D}}\right)=\frac{8}{\pi^{5 / 4} N^{3 / 2}}\left[\frac{\sum n_{k}}{\left(\sum s_{k}\right)^{2}+\left(\sum q_{k}\right)^{2}}\right]^{1 / 2}
$$

where

$n_{k}=n\left(x_{k}, y_{k}\right) \Delta A, s_{k}=\left(x_{k}, y_{k}\right) \Delta A, n_{k}=n\left(x_{k}, y_{k}\right) \Delta A$

The noise elements $n_{k}$ and signal elements, $s_{k}$ and $q_{k}$, are evaluated by numerically integrating Eqs. (12). Standard Mie scattering algorithms are used for the differential cross sections.

To determine the optimum aperture mask a numerical procedure is used to eliminate the set of areas that will maximize the quantity

$$
Q=\frac{\left(\sum s_{k}\right)^{2}+\left(\sum q_{k}\right)^{2}}{\left[n_{k}\right.}
$$

The procedure used is to calculate $Q$ using all the area elements, then eliminate each of the areas, one at a time, and recalculate $Q$. The area element whose elimination gives the greatest increase in $Q$ is included in the aperture mask. This procedure is repeated until no further reduction in the error is possible. The resulting mask is the optimum for the particular distance from the probe volume to the wall.

The calculated masks for the two examples are shown in Fig. 6 . The error bound $\left(\sigma_{f} / f_{D}\right)=0.05$ and 0.002 for the $f / 4$ and $f / 2.5$ examples, respectively. (The noise bandwidth $\Delta f$ was set equal to the Doppler frequency $f_{D}$.) The corresponding SNR $=-7.1 \mathrm{~dB}$ and $25.8 \mathrm{~dB}$, which means that $\mathrm{a}$ counter processor should be a suitable processor for the $f / 2.5$ example, but not for the $f / 4$ example. The mask covers 57 percent of the lens aperture for the f/4 optics and 51 percent for the $f / 2.5$ optics. Also shown on Fig. 6 is the order that the grid elements are blocked in the optimization procedure.

Figure 7 illustrates how the SNR improves as the masks are built up. Note that the SNR improves less quickly as the masks approach their optimum configurations. This is particularly noticeable for the $f / 2.5$ optics where the SNR would only be slightly decreased by using a mask that covers 20 percent of the lens aperture instead of the optimum mask which covers 51 percent.

Figure 8 shows the SNR as a function of distance from the probe volume to the wall (the SNR was calculated for the optimum mask at each location). Note that the SNR reaches a constant value at large distances because of the inclusion of a constant background radiance. This figure illustrates the improvement of the $f / 2.5$ optics compared to the f/4 optics. With f/4 optics, measurements $c$ an be made to about $2 \mathrm{~mm}$ from the wall; while, with $f / 2.5$ optics, measurements $c$ an be made to 
about $0.5 \mathrm{~mm}$. Of course, these results are dependent on the parameters chosen for the examples. In particular, the reflectivity of the wall has a large effect on the SNR for measurements near the wall.

\section{Discussion}

The optical design of a fringe-type laser anemometer for a particular experiment requires several steps to take into account all contraints imposed by the experiment and by the LA system. The initial step is to select a seed material that will follow the flow accelerations and will survive the flow environment. It is usually desirable to use monodisperse particles; however, this may be difficult to achieve in practice.

The next step is to select the fringe spacing and number of fringes. The fringe spacing must be such that the maximum velocity will not result in Doppler frequencies greater than the maximum frequency response of the photodetector and the signal processor. Furthermore, the minimum fringe spacing may be limited by the available solid viewing angle. (The fringe spacing is inversely proportional to the beam crossing angle and hence to the beam separation at the focusing lens.)

Anuther factor that can affect the selection of the fringe spacing is the phase cancellation that occurs when the scattered light is integrated over a large aperture. This can be a significant problem when the fringe spacing is on the order of the particle size. In fact, this phase cancellation is the basis for particle size measurements based on the visibility of the Doppler
burst. $(15,16)$

The selection of the number of fringes is based on several considerations. For counter processors with a given time interval measurement accuracy (typically, 1 ns.), the number of cycles used must be large enough to result in an acceptable error on a single particle measurement. Note that the acceptable single particle measurement error vilj) be larger for highly turbulent

The problem of statistical biasing, which occurs in turbulent flow, may also influence the selection of the number of fringes. For example, the biasing caused by flow angle fiuctuations $(18$ ) is reduced by using a larger number of fringes. Also, the use of frequency shifting can reduce some biasing errors (but at the expense of increasing the required frequency response of the photo detector and signal processor).

After the number and spacing of the fringes have been established, the field stop (pinhole) can be sized to match the probe volume diameter. The focusing lens is selected based on the required working distance and available solid angle. It is generally advantageous to select as fast a lens as is practical.

With the optical system specified, the procedure presented in this paper for determining the optimum aperture mask can be applied. The mask should be optimized for the closest anticipated measurement position to the wall. Based on the calculated SNR, the suitability of a counter processor can be verified.
Note that to apply this procedure the bidirectional reflectivity of the surfaces in the test region must be known. of course, the surfaces should be treated to minimize the reflectivity. (This can be accomplished either by absorbing the light or by reflecting the light in a "safe" direction.) For measurements near the window, the reflectivity of the window must also be known. These reflectivities will generally have to be obtained experimentally. It should be realized that the reflectivities can change during a test run as contaminants accumulate on the walls and windows. In addition, the background radiance due to flames, blackbody radiation, and multiple reflections of the laser light must be estimated or measured.

Finally, it should be noted that the assumption of spherical seed particles that was used for the Mie scattering calculations may introduce some error if the particles are not, in fact, spheres. And, if polydisperse particle size distributions are used, the analysis should be modified to take this into account.

\section{Concluding Remarks}

Using the analysis presented in this paper, the fringe-type laser anemometer can be evaluated in terms of its best possible performance based on the Cramer-Rao bound for the estimate of the variance of the measured Doppler frequency. The relation between this lower bound and the performance of counter-type processors points out the potential performance gain that should be achievable using more optimal processors for low signal-to-noise ratios.

\section{APPENDIX A - CALCULATION OF NOISE}

The irradiance at a point $(x, y)$ on the aperture plane due to wall scatter is obtained by integrating the radiance $L$ of the surface over the solid angle subtended by the image of the pinhole at the point on the aperture (fig. 3). For purposes of calculation, the aperture plane is assumed to be at the plane of the focusing lens instead of the position shown in Fig. 1. The light flux through area $d A$ at point $(x, y)$ on the aperture expressed in photoelectron counts per Doppler cycle is

$n(x, y) d A=$

$\left(\frac{n \lambda}{h c f_{D}}\right) \int_{\Omega^{\prime}}\left[L_{r}\left(x, y ; \theta_{r}, \phi_{r}\right)+L_{b}\right] d \Omega \cos \theta d A$

The radiance due to single scattering of the incident beams from the wall is

$L_{r}\left(x, y ; \theta_{r}, \phi_{r}\right)=\rho^{\prime}\left(x^{\prime}, y^{\prime} ; \theta_{j}, \phi_{j} ; \theta_{r}, \phi_{r}\right) I_{j}\left(x^{\prime}, y^{\prime}\right)$

where $I\left(x^{\prime}, y^{\prime}\right)$ is the irradiance of the beams at the surface and $\rho^{\prime}$ is the bidirectional reflectivity. Note that $\rho^{\prime}$ is a function of both the direction of incident and the reflected light.

If the wall is normal to the optical axis, the irradiance of the Gaussian beams at the surface is 


$$
\begin{aligned}
I_{i}\left(x^{\prime}, y^{\prime}\right) & =\frac{P}{\pi}\left\{\frac{1}{w^{2}\left(z_{1}\right)} e^{-\frac{2\left(x_{1}^{2}+y_{1}^{2}\right)}{w^{2}\left(z_{1}\right)}}\right. \\
& \left.+\frac{1}{w^{2}\left(z_{2}\right)} e^{-\frac{2\left(x_{2}^{2}+y_{2}^{2}\right)}{w^{2}\left(z_{2}\right)}}\right\}
\end{aligned}
$$

where $\left(x^{\prime}, y^{\prime}\right)$ is the point on the wall along the ray through point $(x, y)$ in the direction $(\theta, \phi)$.

Also, $\left(x_{1}, y_{1}, z_{1}\right)$ and $\left(x_{2}, y_{2}, z_{2}\right)$ are the coordinates of the point on the wall in coordinate system with $z_{1}$ and $z_{2}$ along the beam propagation directions and are given by

$\left.\begin{array}{l}x_{1,2} \\ y_{1,2} \\ z_{1,2}\end{array}\right]=\left[\begin{array}{ccc}\cos \beta & 0 & \pm \sin \beta \\ 0 & 1 & 0 \\ \mp \sin \beta & 0 & \cos \beta\end{array}\right]\left[\begin{array}{l}x^{\prime} \\ y^{\prime} \\ d^{\prime}\end{array}\right.$

with $d$ being the distance from the focal point to the wall and $w(z)$ the beam radius given by

$$
w(z)=w_{0}\left[1+\left(\frac{\lambda z}{\pi w_{0}^{2}}\right)^{2}\right]^{1 / 2}
$$

APPENDIX B - CALCULATION OF SIGNAL

The signal is calculated using Mie scattering theory, (19) which takes into account both the variation in the amplitude and phase of the scattered fields as a function of scattering angle. The incident beams are located in the $x-z$ plane and have a crossing angle $2 B$ ( $f$ ig. 4 ).

The electric fields of the incident beams near the waists are represented as plane waves

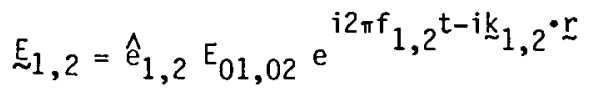

where the field amplitudes are $E_{01,02}=\left(2 Z_{0}\right)^{1 / 2} I_{0}$; with $I_{0}$ being the peak irradiance of each beam at its waist, which is related to the laser power and waist diameter by $\mathrm{I}_{0}=\mathrm{P}_{\ell} / \pi \mathrm{W}_{0}$. The electric fields of the beams are assumed to be plane polarized with unit vectors $\hat{e}_{1}$ and $\hat{e}_{2}$.

We define the following unit vectors:

$$
\begin{gathered}
\hat{s}_{1,2}=\left(k_{s} \times k_{1,2}\right) /\left|k_{s} \times k_{1,2}\right| \\
\hat{p}_{1,2}=\left(k_{1,2} \times \hat{s}_{1,2}\right) / k \\
\hat{a}_{1,2}=\left(k_{s} \times \hat{s}_{1,2}\right) / k
\end{gathered}
$$

where $\hat{s}_{1}$ and $\hat{s}_{2}$ are normal to the scattering planes; $\hat{p}_{1}$ and $\hat{p}_{2}$ are normal to the propagation directions of the incident beams and in the scattering planes; and, $\mathrm{q}_{1}$ and $\mathrm{q}_{2}$ are normal to the scattered wave propagation direction and in the scattering planes.
The incident fields can be expressed in terms of the components in the scattering plane and normal to the scattering plane

$$
E_{1,2}=E_{1 s, 2 s} \hat{s}_{1,2}+E_{1 p, 2 p} \hat{p}_{1,2}
$$

The scattered fie]ds are given by Mie scattering theory for spheres (19)

$$
\begin{aligned}
E_{1,2}^{(s)} & =\frac{i e^{-i k R}}{k R}\left[E_{1 s, 2 s} s_{s}\left(\theta_{1,2}\right) \hat{s}_{1,2}\right. \\
& \left.+E_{1 p, 2 p} s_{p}\left(\theta_{1,2}\right) \hat{a}_{1,2}\right]
\end{aligned}
$$

where $R$ is the distance from the scatterer to the observation point, and $S_{S}(\theta)$ and $S_{p}(\theta)$ are the usual Mie scattering amplitude functions with the scattering angles given by

$$
\cos \theta_{1,2}=\left(k_{1,2} \cdot k_{s}\right) / k^{2}
$$

The irradiance of the scattered light at the observation point is

$I_{s}(x, y)=\left|E_{1}^{(s)}+E_{2}^{(s)}\right|^{2} / 2 Z_{o}=I_{s d c}+I_{s a c}$

This is composed of the sum of a slowly varying de component, I $s d c$, corresponding to the pedestal of the Doppler burst and an ac component, $I_{\mathrm{sac}}$, modulated at the Doppler frequency. These can be written as

$$
\begin{aligned}
& I_{s d c}(x, y)=I_{0}\left[\left(\hat{e}_{1} \cdot \hat{s}_{1}\right)^{2} \sigma_{s}\left(\theta_{1}\right)+\left(\hat{e}_{1} \cdot \hat{p}_{1}\right)^{2} \sigma_{p}\left(\theta_{1}\right)\right. \\
& \left.+\left(\hat{e}_{2} \cdot \hat{s}_{2}\right)^{2} \sigma_{s}\left(\theta_{2}\right)+\left(\hat{e}_{2} \cdot \hat{p}_{2}\right)^{2} \sigma_{p}\left(\theta_{2}\right)\right] \\
& I_{s a c}(x, y)=I_{0} \sum_{\alpha=s, p} \sigma_{\alpha \beta} \\
& \left(\cos \delta_{\alpha \beta} \cos 2 \pi f_{D} t-\sin \delta_{\alpha \beta} \sin 2 \pi f_{D} t\right)
\end{aligned}
$$

Note that $I_{S a c}$ is expressed in a quadrature representation form. Because the phase of I $\mathrm{sac}$ varies across the aperture, the detected signal will, in general, be less than the signal that would be detected if the phase were constant.

The Mie complex amplitude functions can be written as

$$
S_{\alpha}(\theta)=\left|S_{\alpha}(\theta)\right| e^{i \delta_{\alpha}(\theta)}, \alpha=S, p
$$

The differential cross sections for "s" and "p" scattering are

$$
\sigma_{\alpha}(\theta)=(\lambda / 2 \pi) \text { is }(\theta) 1^{2}, \alpha=s, p
$$

In Eq. (B10) we also introduced the following

$\sigma_{s s}=\left[\sigma_{s}\left(\theta_{1}\right) \sigma_{s}\left(\theta_{2}\right)\right]^{1 / 2}\left(\hat{e}_{1} \cdot \hat{s}_{1}\right)\left(\hat{e}_{2} \cdot \hat{s}_{2}\right)\left(\hat{s}_{1} \cdot \hat{s}_{2}\right)$ 


$$
\begin{gathered}
\sigma_{p p}=\left[\begin{array}{lll}
\sigma_{p}\left(\theta_{1}\right) & \sigma_{p}\left(\theta_{2}\right)
\end{array}\right]^{1 / 2}\left(\hat{e}_{1} \cdot \hat{p}_{1}\right)\left(\hat{e}_{2} \cdot \hat{p}_{2}\right)\left(\hat{q}_{1} \cdot \hat{q}_{2}\right) \\
\sigma_{s p}=\left[\begin{array}{lll}
\sigma_{s}\left(\theta_{1}\right) & \sigma_{p}\left(\theta_{2}\right)
\end{array}\right]^{1 / 2}\left(\hat{e}_{1} \cdot \hat{s}_{1}\right)\left(\hat{e}_{2} \cdot \hat{p}_{2}\right)\left(\hat{s}_{1} \cdot \hat{\theta}_{2}\right) \\
\sigma_{p s}=\left[\begin{array}{ll}
\sigma_{p}\left(\theta_{1}\right) & \sigma_{s}\left(\theta_{2}\right)
\end{array}\right]^{1 / 2}\left(\hat{e}_{1} \cdot \hat{p}_{1}\right)\left(\hat{e}_{2} \cdot \hat{s}_{2}\right)\left(\hat{a}_{1} \cdot \hat{s}_{2}\right) \\
\delta_{\alpha \beta}=\delta_{\alpha}\left(\theta_{1}\right)-\delta_{\beta}\left(\theta_{2}\right), \quad \alpha, \beta=s, p \quad(B 13) \\
\text { References }
\end{gathered}
$$

\section{References}

1. Schodl, R., "Laser Dual-Beam Method for Flow Measurements in Turbomachines," ASME Paper 74-GT-157, Mar. 1974.

2. Lading, L., "Estimating Time and Time-Lag in Time-of-Flight Velocimetry," Applied Optics, Vol. 22, No. 22, Nov. 15, 1983, pp. 3637-3643.

3. Goldman, L. J., and Seasholtz, R. G., "Comparison of Laser Anemometer Measurements and Theory in an Annular Turbine Cascade with Experimental Accuracy Determined by Parameter Est imation," Engineering Applications of Laser Velocimetry, edited by H. W. Coleman and P. A. Pfund, ASME, 1982 , pp. 83-92.

4. Goldman, L. J., and Seasholtz, R. G., "Laser Anemometer Measurements in an Annular Cascade of Core Turbine Vanes and Comparison with Theory," NASA TP-2018, 1982.

5. Powell, J. A., Strazisar, A. J., and Seasholtz, R. G., "High-Speed Laser Anemometer System for Intrarotor Flow Mapping in Turbomachinery," NASA TP-1663, 1982.

6. Powel1, J. A., Strazisar, A. J., and Seasholtz, R. G., "Efficient Laser Anemometer for Intra-Rotor Flow Mapping in Turbomachinery," Journal of Engineering for Power, Vol. 103, No. 2, Apr. 1981, pp. 424-4239.

7. Gardavsky, J., and Kleine, R., "Reflection Suppression by Polarization in Backscatter LDA Measurements Near Walls and in Two-Phase Flows," Applied Optics, Vol. 20., No. 23, Dec. 1, 1981, pp. 4110-4123.

8. Gardavsky, J., and Bok, J., "Scattering of Circularly Polarized Light' in Laser Doppler Anemometry," Applied Optics, Vol. 21, No.1,
9. Mishina, H., Vlachos, N. S., and Whitelaw, J. H., "Effect of Wall Scattering on SNR in Laser Doppler Anemometry," Applied Optics, Vol. 18, July 15, 1979, pp. 2480-2485.

10. Mishina, H., Takahashi, K., and Asakura, T., "Effect of Wall Scattering on SNR in Off-Axis Differential-Type Laser Doppler Velocimetry," Optical and Quantum Electronics, Vol. 15, Sept. 1983, pp. 419-431.

11. Eckbreth, A. C., and Davis, J. W., "Spatial Resolution Enhancement in Coaxial Light Scattering Geometries - Raman Laser Diagnostics," Applied Optics, Vol. 16, Apr. 1977, pp. 804-806.

12. Whalen, A. D., Detection of Signals in Noise, Academic Press, New York, 19/1, pp. 324-331.

13. Lading, L., and Jensen, A. S., "Estimating the Spectral Width of a Narrowband Optical Signal," Applied Optics, Vol. 19, Aug. 15, 1980 , pp. 2750-2756.

14. Adrian, R. J., Humphrey, J. A. C., and Whitelaw, J. H., "Frequency Measurement Errors Due to Noise in LDV Signals," in The Accuracy of Flow Measurements by Laser Doppler Methods, edited by P. Buchiave, J. M. Delhaye, F. Durst, W. K. George, K. Refslund, and J. H. Whitelaw, Proceedings in LOA-Symposium Copenhagen, Skovlunde, Denmark, 1976, pp. 287-311.

15. Chu, W. P., and Robinson, D. M., "Scattering from a Moving Spherical Particle by Two Crossed Coherent Plane Waves, "Applied 0ptics, Vol. 16, Mar. 1977, pp. 619-626.

16. Adrian, R. J., and Orloff, K. L., "Laser Anemometer Signals: Visibility Characteristics and Application to Particle Sizing," Applied Optics, Vol. 16, Mar. 1977, pp. 677-684.

17. Edwards, R. V., and Jensen, A. S., "ParticleSampling Statistics in Laser Anemometers Sample-and-Hold Systems and Saturable Systems," Journal of Fluid Mechanics, Vol. 133, Aug. 1983, pp. 397-411.

18. Seasholtz, R. G., "Laser Doppler, Velocimeter System for Turbine Stator Cascade Studies and Analysis of Statistical Biasing Errors," NASA TN 0-8297, 1977.

19. Van de Hulst, H. C., Light Scattering by Small Particles, Dover Publications, New York, 1981, Chapter 9 . 


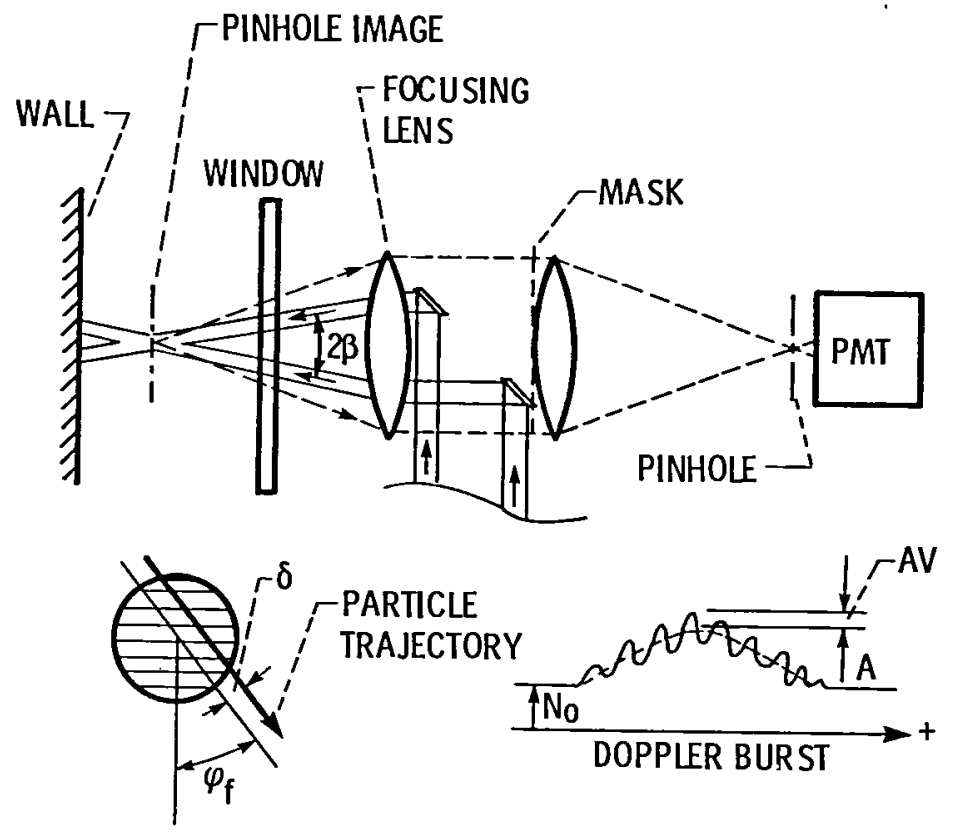

Figure 1. - Optical configuration of coaxial backscatter fringetype laser anemometer.

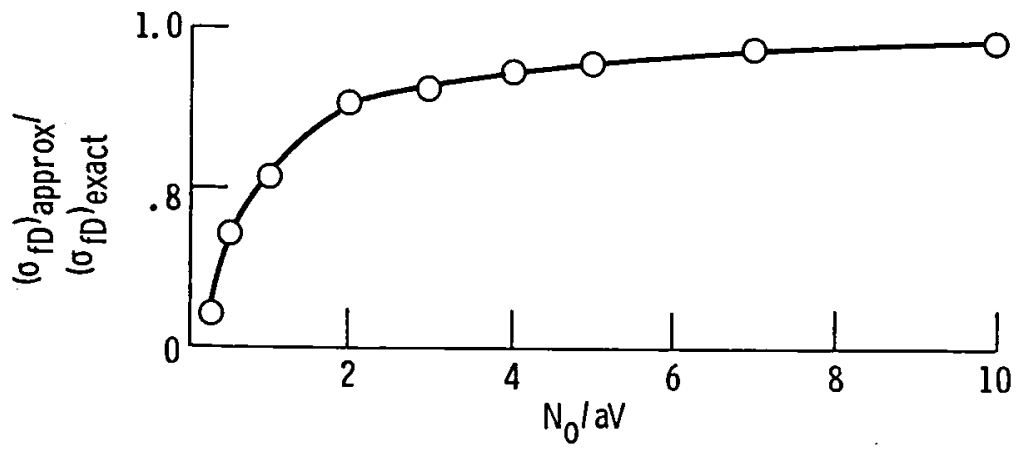

Figure 2. - Ratio of approximate value (eq. 9) to exact value (eq. 3) of lower bound for relative error of Doppler frequency versus ratio of background amplitude to signal amplitude: Parameters used in calculation are: number fringes, $\mathrm{N}=10$; signal amplitude, $\mathrm{aV}=50$ counts/Doppler cycle; visibility, $V=1$; and, phase, $4=0$. 


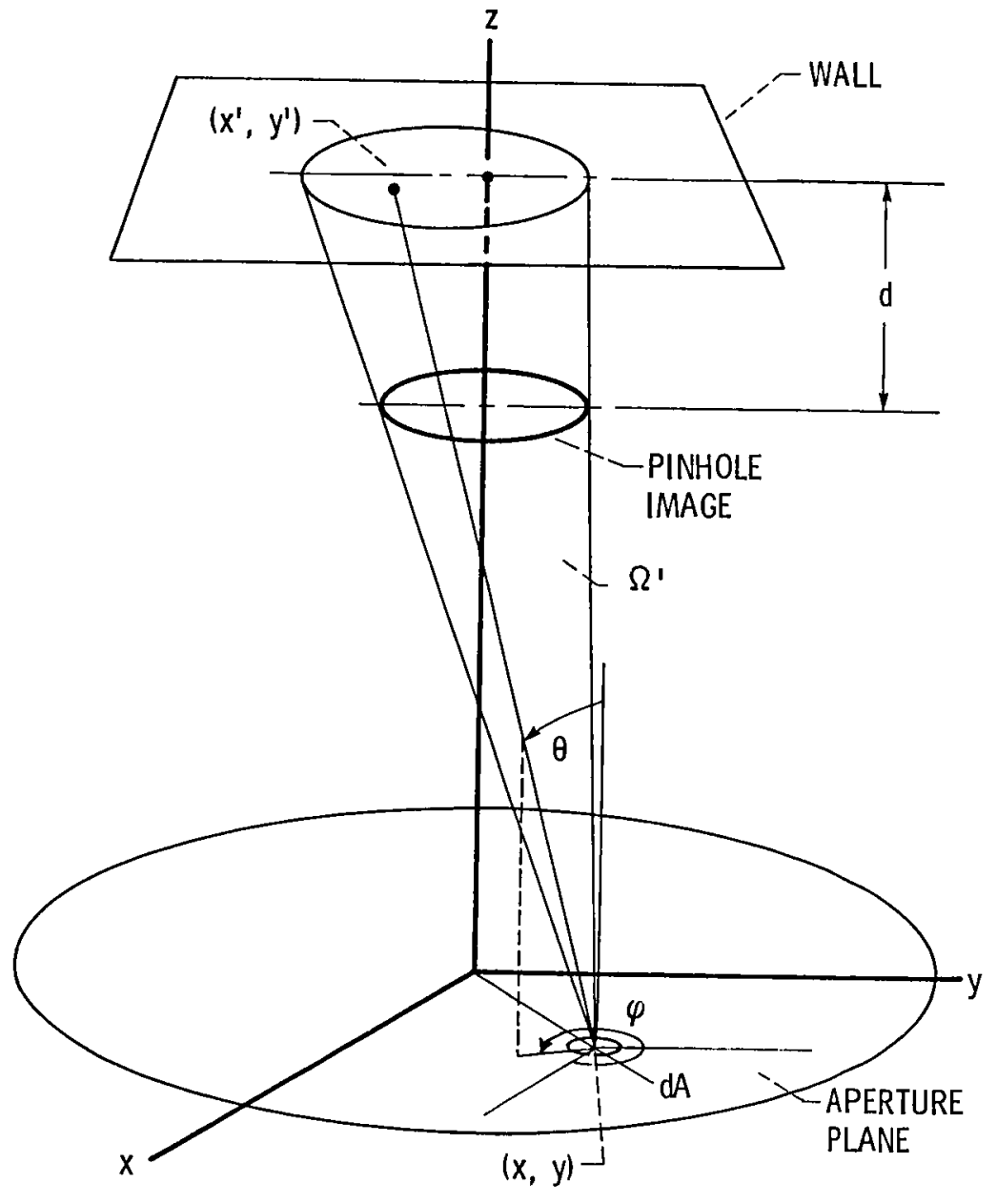

Figure 3. - Geometry used for evaluation of wall scatter noise.

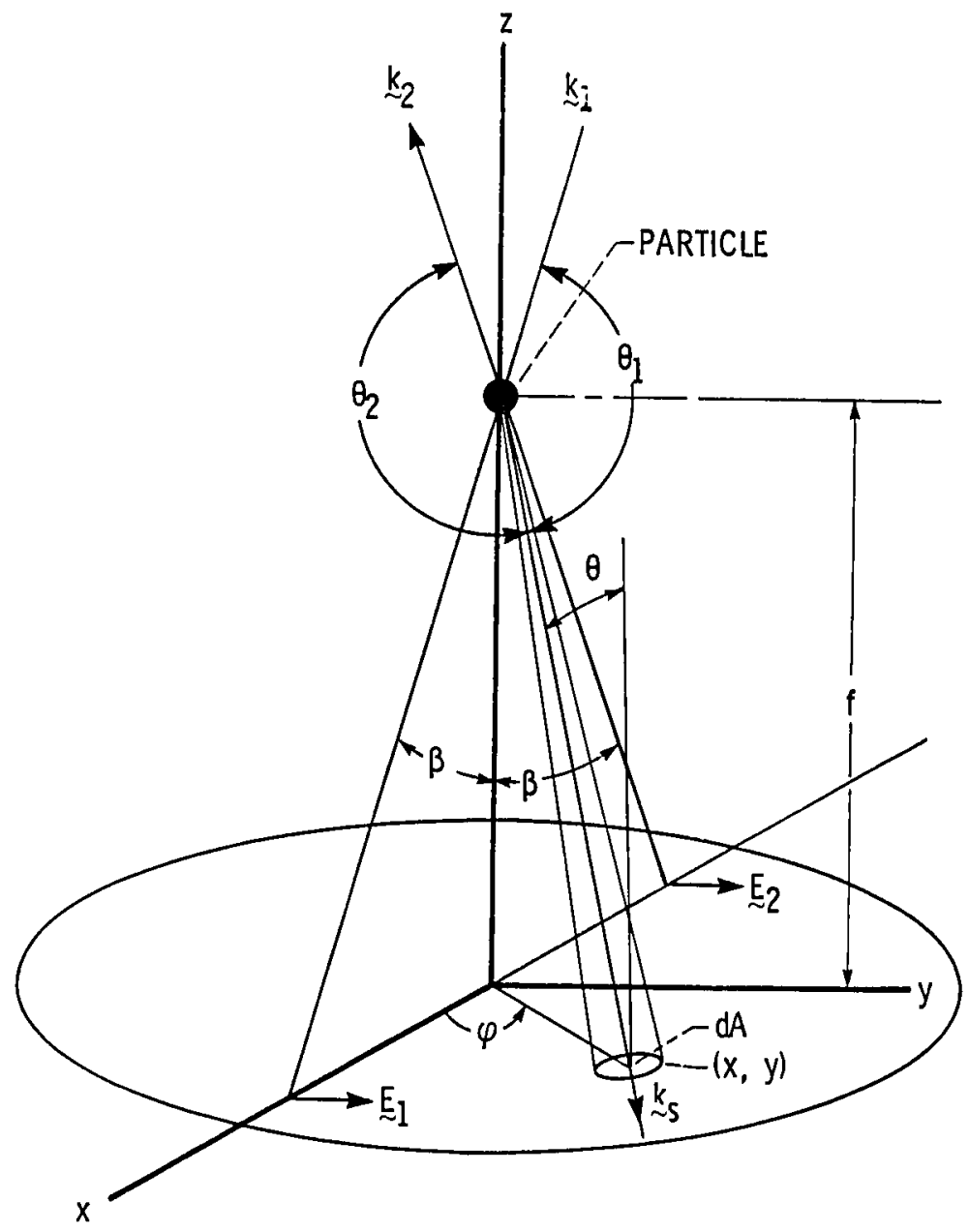

Figure 4. - Geometry used for evaluation of Mie scattering. 

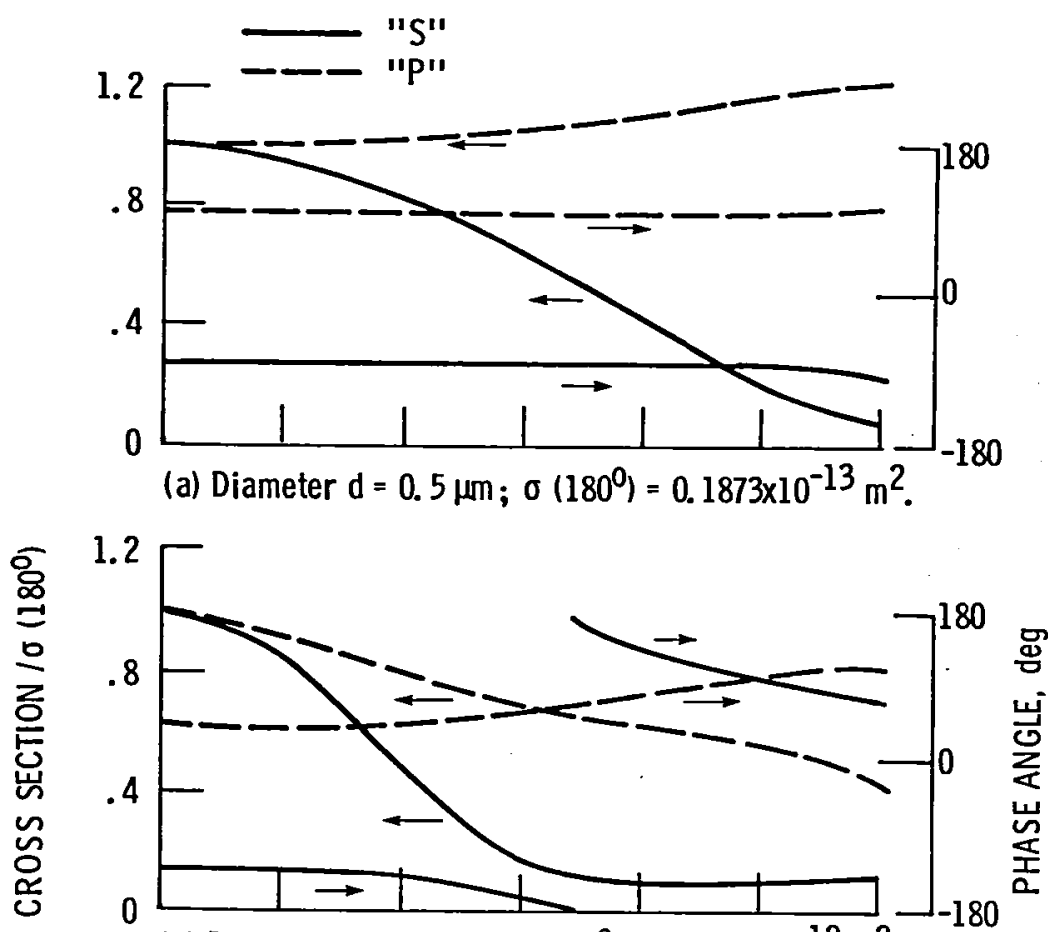

(b) Diameter $d=1.0 \mu \mathrm{m} ; \sigma\left(180^{\circ}\right)=0.3695 \times 10^{-12} \mathrm{~m}^{2}$.

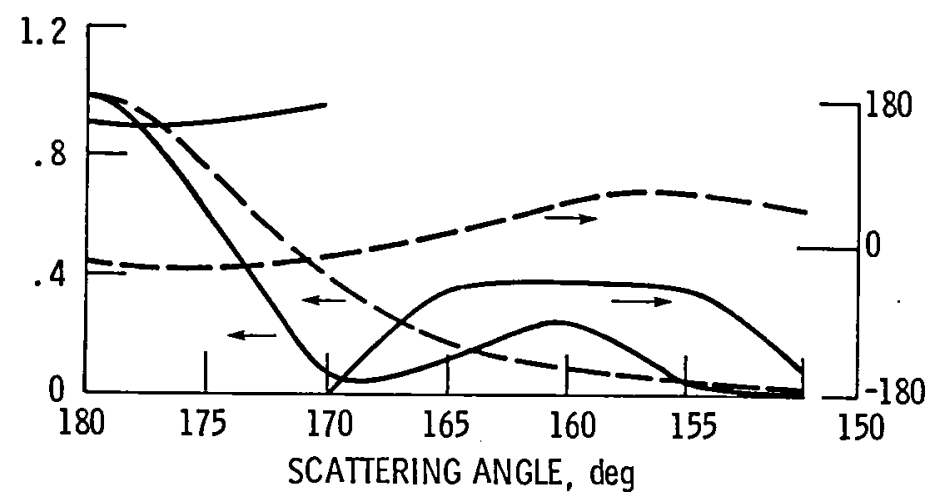

(c) Diameter $d=1.5 \mu \mathrm{m} ; \sigma\left(180^{\circ}\right)=0.2169 \times 10^{-11} \mathrm{~m}^{2}$.

Figure 5. - Differential scattering cross section and phase versus scattering angle for polystyrene spheres $(n=1.59)$. Wavelength $\lambda=0.5145 \mu \mathrm{m}$. 


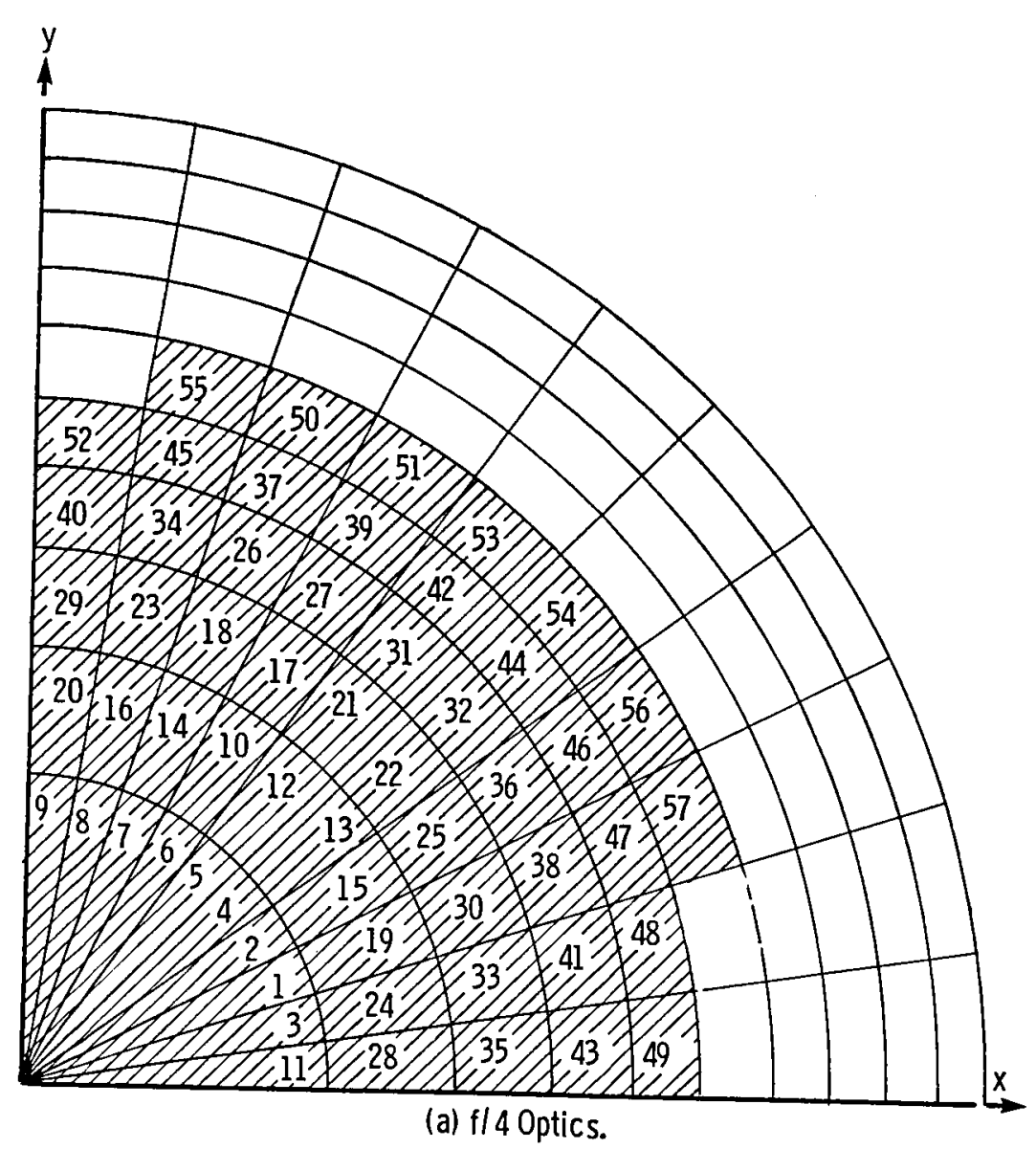

Figure 6. - Optimum masks for probe volume located $1 \mathrm{~mm}$ from wall. Grid elements are numbered in the order that the mask is built up.

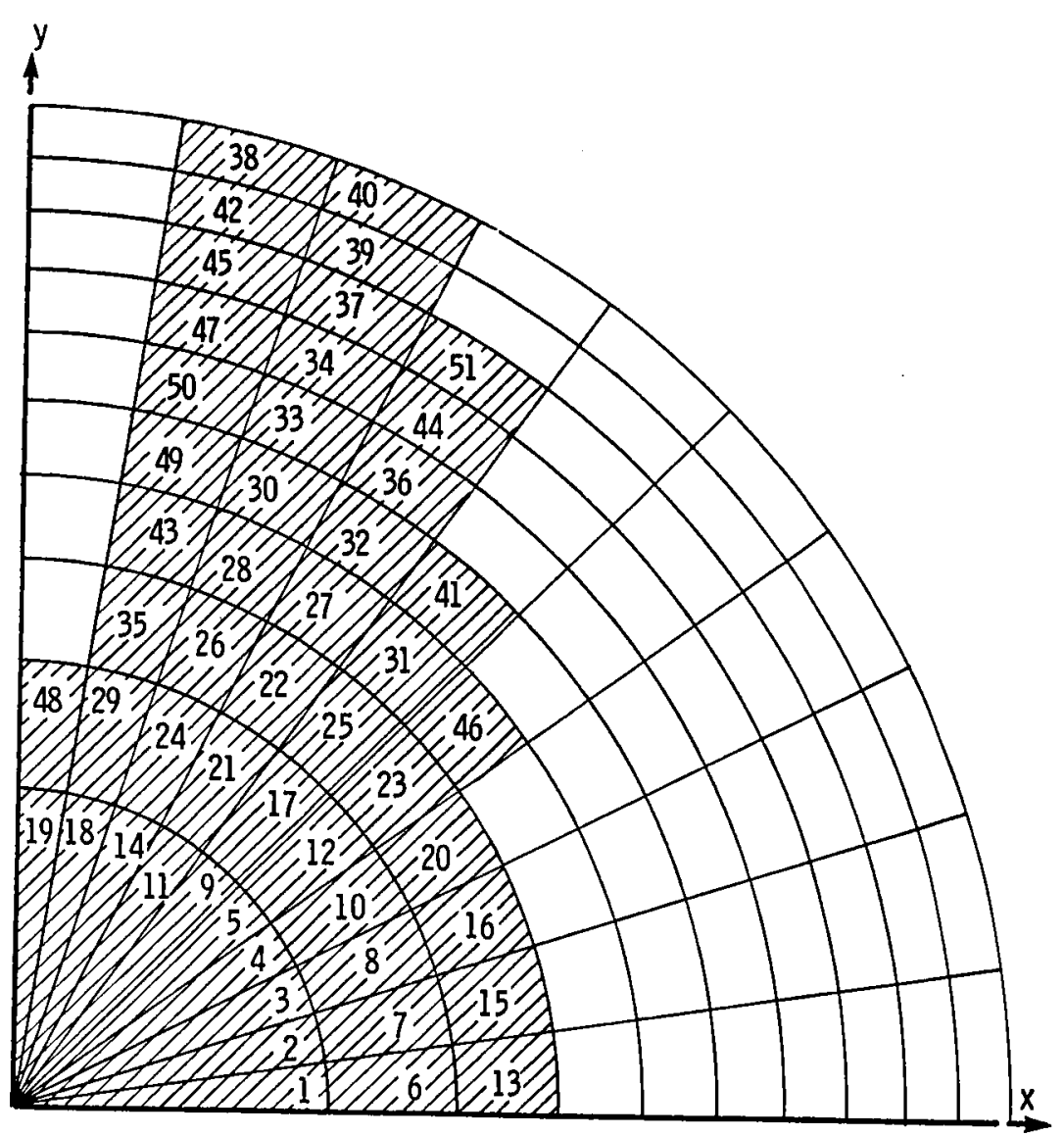

(b) f/2.5 Optics.

Figure 6. - Concluded. 

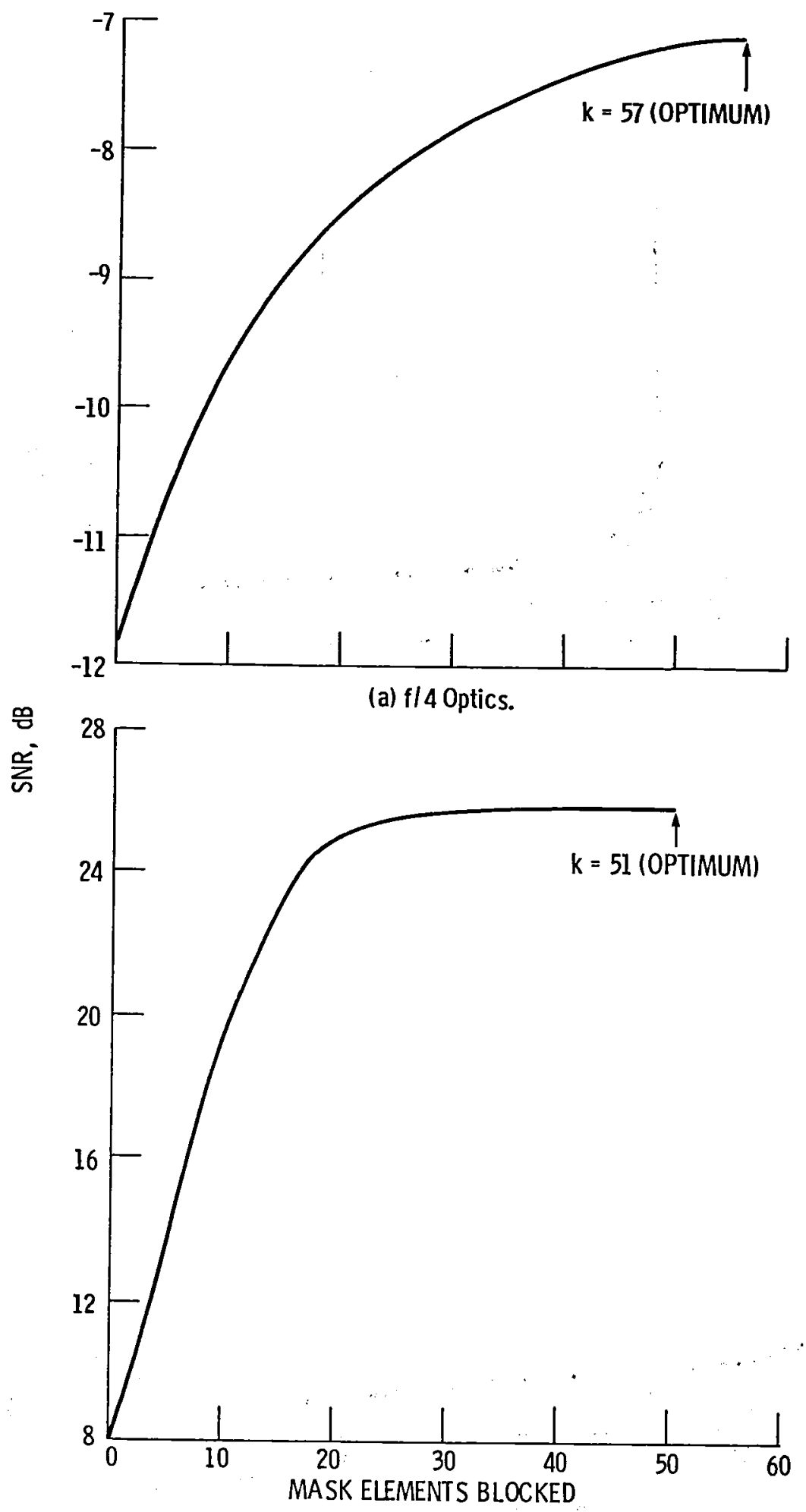

(b) f/2.5 Optics.

Figure 7. - Signal-to-noise ratio (SNR) versus accumulation of mask area elements. 


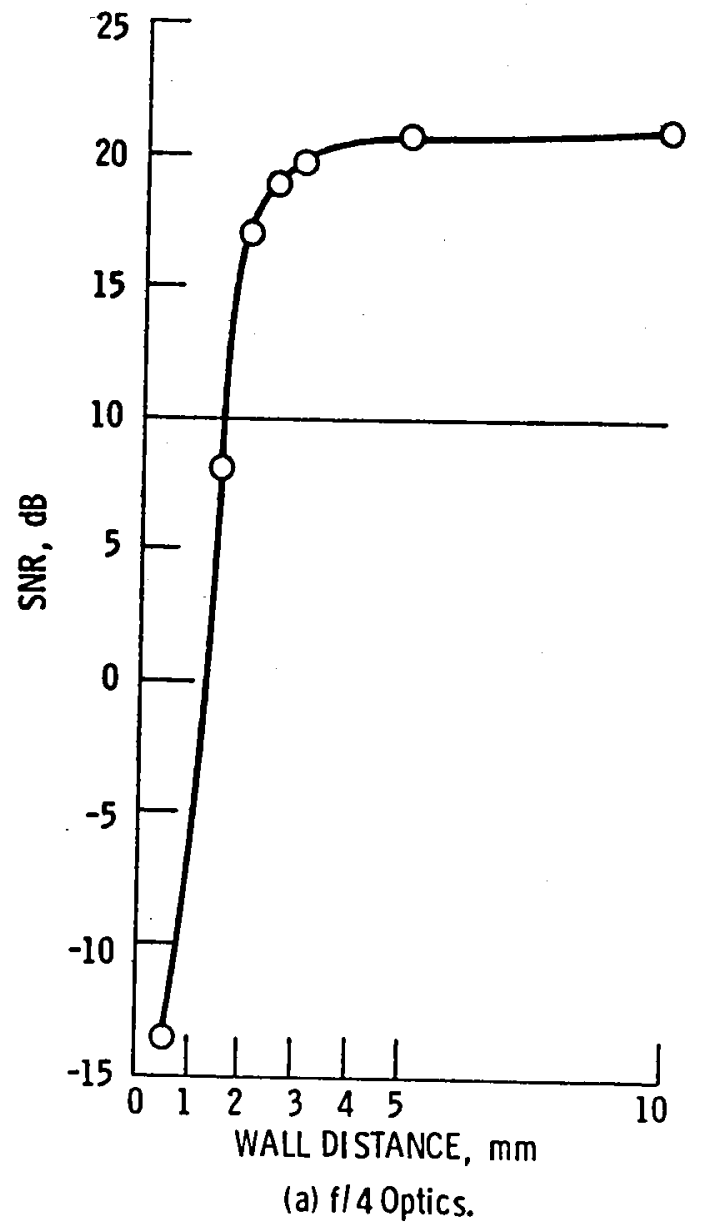

Figure 8. - Signal-to-noise ratio (SNR) for optimum mask versus distance of probe volume from wall.

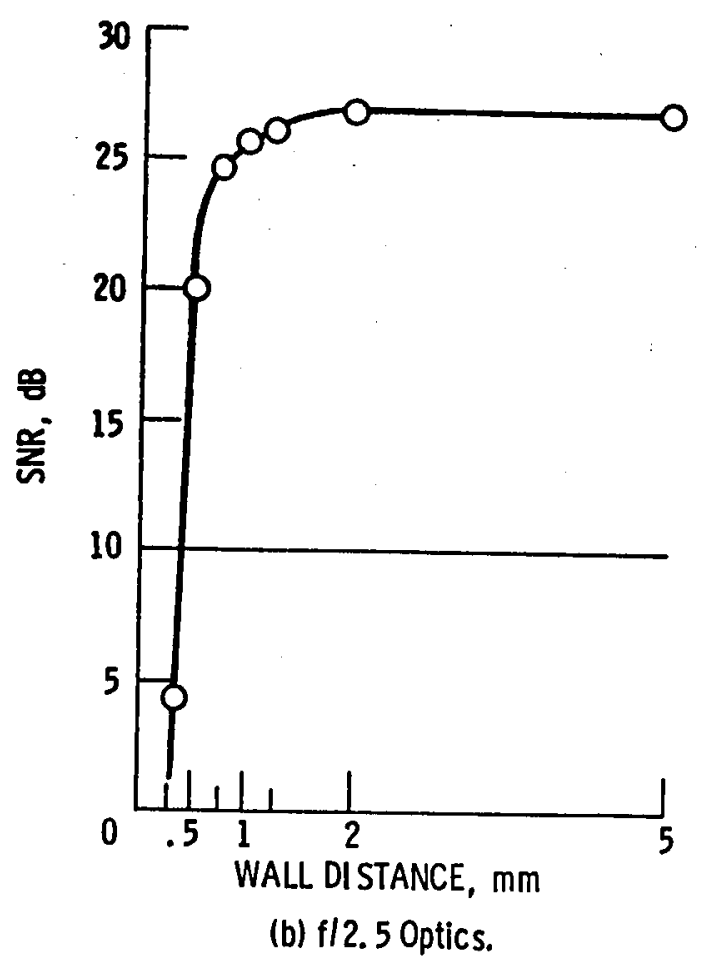

Figure 8. - Concluded 


\begin{tabular}{|c|c|c|c|}
\hline \multirow{2}{*}{\multicolumn{2}{|c|}{$\begin{array}{l}\text { 1. Report No. NASA TM-83658 } \\
\text { AIAA-84- } 7459\end{array}$}} & \multicolumn{2}{|c|}{ 3. Reciplent's Catalog No. } \\
\hline & & \multicolumn{2}{|l|}{ 5. Report Date } \\
\hline \multicolumn{2}{|c|}{$\begin{array}{l}\text { 7. Author(s) } \\
\text { Richard G. Seasholtz, Lawrence G. Oberle, and } \\
\text { Donald H. Heikle }\end{array}$} & \multicolumn{2}{|c|}{$\begin{array}{l}\text { 8. Performing Organization Report No. } \\
\text { E-2099 }\end{array}$} \\
\hline \multicolumn{2}{|c|}{$\begin{array}{l}\text { 9. Performing Organization Name and Address } \\
\text { National Aeronautics and Space Administration } \\
\text { Lewis Research Center } \\
\text { Cleveland, Ohio } 44135\end{array}$} & \multicolumn{2}{|c|}{\begin{tabular}{|l} 
11. Contract or Grant No. \\
\end{tabular}} \\
\hline \multirow{2}{*}{\multicolumn{2}{|c|}{$\begin{array}{l}\text { 12. Sponsoring Agency Name and Address } \\
\text { National Aeronautics and Space Administration } \\
\text { Washington, D.C. } 20546\end{array}$}} & \multicolumn{2}{|c|}{$\begin{array}{l}\text { 13. Type of Report and Period Covered } \\
\text { - Technical Memorandum }\end{array}$} \\
\hline & & \multicolumn{2}{|c|}{ 14. Sponsoring Agency Code } \\
\hline \multicolumn{4}{|c|}{$\begin{array}{l}\text { 15. Supplementary Notes } \\
\text { Prepared for the Twentieth Joint Propulsion Conference cosponsored by the AIAA, } \\
\text { SAE, and ASME, Cincinnati, Ohio, June } 11-13,1984 \text {. }\end{array}$} \\
\hline \multicolumn{4}{|c|}{$\begin{array}{l}\text { 16. Abstract } \\
\text { The fringe-type laser anemometer is analyzed using the Cramer-Rao bound for the } \\
\text { variance of the estimate of the Doppler frequency as a figure-of-merit. Mie } \\
\text { scattering theory is used to calculate the Doppler signal wherein both the } \\
\text { amplitude and phase of the scattered light are taken into account. The noise } \\
\text { from wall scatter is calculated using the wall bidi rectional reflectivity and } \\
\text { the irradiance of the incident beams. A procedure is described to determine the } \\
\text { optimum aperture mask for the probe volume located a given distance from a wall. } \\
\text { The expected performance of counter-type processors is also discussed in } \\
\text { relation to the Cramer-Rao bound. Numerical examples are presented for a } \\
\text { coaxial backscatter anemometer. }\end{array}$} \\
\hline 17. Key Words (Suggested by Author(s)) & \multirow{2}{*}{\multicolumn{3}{|c|}{$\begin{array}{l}\text { 18. Distribution Statement } \\
\text { Unclassified - unlimited } \\
\text { STAR Category } 35\end{array}$}} \\
\hline $\begin{array}{l}\text { Laser anemometers } \\
\text { Laser velocimeters }\end{array}$ & & & \\
\hline $\begin{array}{l}\text { 19. Security Classif. (of this report) } \\
\text { Unclass i fied }\end{array}$ & $\begin{array}{l}\text { 0. Security Classif. (of this page) } \\
\text { Unclassified }\end{array}$ & 21. No. of pages & 22. Price* \\
\hline
\end{tabular}

"For sale by the National Technical Information Service, Springfield, Virginia 22161 
National Aeronautics and Space Administration BOOK

Washington, D.C.

20546

Official Business

Penalty for Private Use, $\$ 300$

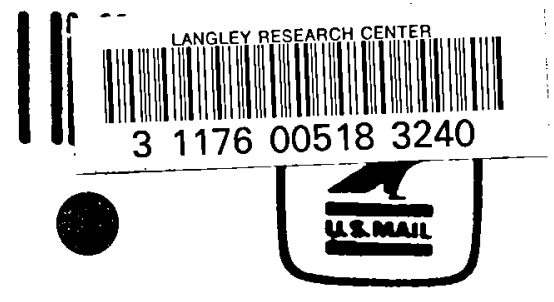

Pontege and Fees Paid Netional Aeroneutics and Spece Administration NASA-45I 